\title{
A New Physical Performance Classification System for Elite Handball Players: Cluster Analysis
}

\author{
by \\ Iker J. Bautista ${ }^{1}$, Ignacio J. Chirosa², Joseph E. Robinson ${ }^{2}$, . Roland van der Tillaar ${ }^{3}$, \\ Luis J. Chirosa², Isidoro Martínez Martín ${ }^{4}$
}

The aim of the present study was to identify different cluster groups of handball players according to their physical performance level assessed in a series of physical assessments, which could then be used to design a training program based on individual strengths and weaknesses, and to determine which of these variables best identified elite performance in a group of under-19 [U19] national level handball players. Players of the U19 National Handball team ( $n=16)$ performed a set of tests to determine: $10 \mathrm{~m}$ (ST10) and $20 \mathrm{~m}$ (ST20) sprint time, ball release velocity (BRv), countermovement jump (CMJ) height and squat jump (SJ) height. All players also performed an incremental-load bench press test to determine the 1 repetition maximum (1RMest), the load corresponding to maximum mean power $\left(\operatorname{Load}_{M P}\right)$, the mean propulsive phase power at LoadMP (PMPPMP) and the peak power at LoadMP (PPEAKMP). Cluster analyses of the test results generated four groupings of players. The variables best able to discriminate physical performance were BRv, ST20, 1RMest, $P_{P E A K M P}$ and $P_{M P P M P}$. These variables could help coaches identify talent or monitor the physical performance of athletes in their team. Each cluster of players has a particular weakness related to physical performance and therefore, the cluster results can be applied to a specific training programmed based on individual needs.

Key words: strength, sport, physical fitness.

\section{Introduction}

Given the types of intermittent maximum effort needed during a team sport such as handball, it is important to evaluate performance by measuring variables such as muscle strength, velocity and resistance (Marques, 2010; Michalsik et al., 2013; Póvoas et al., 2014). The factors that determine performance in handball are explosive actions such as jumping (i.e., vertical or horizontal), speed of reduced-space displacements (i.e., 10 and $20 \mathrm{~m}$ sprint time) and ball release velocity (BRv). To assess physical performance of a handball player, several tests are commonly used encompassing sprinting, jumping, agility and maximal strength as often measured in the one repetition maximum (1RM), or ball release velocity tests (Marques and González-Badillo, 2006). These tests have two main objectives. Thus, according to the scores obtained in these tests, athletes can be classified in terms of a particular skill (e.g., the player who is able to throw the ball faster or lift the heaviest weight in the bench press) (Ingebrigtsen et al., 2013; Vila et al., 2012). The problem with this type of classification is that, despite an existing correlation between the maximum weight lifted in the bench press and ball throwing velocity

\footnotetext{
1 - FisioSalud Elite, Health, Training \& Innovation

2 - Department of Physical Education and Sport. University of Granada (Spain).

3 - Faculty of Teacher Education of Nord Trøndelag University College. Department of Teacher Education and Sports (Levanger, Norway).

4- Department of Physical Education and Sport. University of Leon (Spain).
} 
(Gorostiaga et al., 2005; Granados et al., 2007; Marques et al., 2007), the player who throws the ball more rapidly will not necessarily be the individual attaining the highest 1RM in the bench press. The second objective of such tests is to compare the effects of training programs on performance during a given period of time (Gorostiaga et al., 1999) or during a competition season (Bresciani et al., 2010; Gorostiaga et al., 2005; Granados et al., 2008).

A search of the scientific literature indicates that such measures of performance are commonly used to determine the response to a given training intervention (Chelly et al., 2009, 2013; Hermassi et al., 2011). The researcher looks for differences between pre- and post test values of a certain variable. Moreover, test scores may be used to measure the effect size produced by a training intervention on a study population (Peterson et al., 2004; Rhea et al., 2003). When used in this manner, the cross-sectional perspective of these tests is completely lost. In other words, tests are not used to order players according to their performance capacity. This information could be of vital importance to measure the physical state of a player before a training program compared with the rest of the players. A further use of a physical performance test is the identification of young talent. This line of research has generated many studies in different sports such as soccer (Unnithan et al., 2012; Votteler and Höner, 2013; William and Reilly, 2000), handball (Baker et al., 2013; Lidor et al., 2005; Mohamed et al., 2009), basketball (Hoare, 2000) or rugby (Gabbett, 2006, 2011) in which the principle objective was to differentiate elite from amateur players based on variables related to physical performance, anthropometric measures or decision making ability. In a recent study, Matthys et al. (2011) developed a multidisciplinary model to identify talented young handball players (under-14 [U14], under-16 [U16] and under-18 [U18]). The variables included in the model were anthropometric, physical performance and psychological factors. Results indicated that elite players could be distinguished from non-elite ones according to physical performance characteristics, although the discrimination capacity of this method varied according to the age group tested.

However, in the research conducted by
Matthys et al. (2011) clustering of handball players occurred naturally depending upon their respective team (elite vs. non-elite team) and age (U14, U16 and U18). Using multivariate statistics (i.e., cluster analysis) and theoretical knowledge about performance, it is possible to create an objective physical performance classification system of the handball players. Therefore, the aim of the present study was to identify different cluster groups of handball players according to their physical performance level evaluated in a series of physical assessments (a countermovement jump [CMJ], a squat jump [SJ], $10 \mathrm{~m}$ sprint time [ST10], $20 \mathrm{~m}$ sprint time [ST 20 ], the one repetition maximum [1RMest], peak power at the propulsive phase $\left[P_{P E A K M P}\right]$ and mean power at the propulsive phase [PMPPMP] in the bench press and ball release velocity [BRv]), which could then be used to design a training program based on individual strengths and weaknesses, and to determine which of these variables best identified elite performance in a group of under-19 [U19] national level handball players.

\section{Material and Methods}

\section{Participants}

The study participants were 16 male players in the U19 National Handball Team. Participants' age, body mass and height (mean \pm SD) were $18 \pm 0.4$ years, $87.38 \pm 9.97 \mathrm{~kg}$ and $189 \pm$ $6.72 \mathrm{~cm}$, respectively. All the players had at least two years of experience in strength training. Written informed consent was obtained from the participants after the nature of the study and its objectives had been explained. The study protocol received University of Granada Review Board approval and was performed in accordance with the tenets of the Declaration of Helsinki.

\section{Testing procedures}

A cross-sectional design was performed to measure physical performance variables in young handball players. This investigation was conducted one month prior to the European Junior Handball Championship in 2013. Using a multivariate approach, a hierarchical cluster analysis was performed to create a grouping of training based on different important training variables in handball players. After that, a descriptive discriminant analysis was performed to indicate the most important variable to generate this grouping classification. The 
measured variables included in the analysis were in vertical dimension (CMJ and SJ), in horizontal dimension (ST10 and $\mathrm{ST}_{20}$ ) and cross-sectional dimension (1RMest, PPмpPMP and PMPPMP in the bench press and BRv).

The physical tests were performed during the training camp for the European Junior Handball Championship in 2013. Sprint time, $\mathrm{BRv}, \mathrm{CMJ}$, SJ and incremental-load bench press tests were applied since these are regularly used in testing physical performance in handball and measure variables such as muscle strength, power and velocity in the upper and lower body very well (Granados et al., 2008; Ingebrigtsen et al., 2013; Krüger et al., 2014; Marques and GonzálezBadillo, 2006). These physical performance tests were conducted at the team's playing court and training facilities. Before the tests, participants attended a familiarization session in which each test protocol and its key points were explained in detail.

All participants undertook their standard 15 min warm up protocol involving sprints with direction changes and ball passes. After a $5 \mathrm{~min}$ rest period in which the subjects could drink water ad libitum, they completed the physical performance assessment tests established for the session. The tests were performed over 3 days as follows: Day 1, ST10 and ST20 and BRv; Day 2, CMJ and SJ; and Day 3, an incremental-load bench press test. All tests were executed before the regular handball training session. At least $24 \mathrm{~h}$ elapsed between each testing session.

Sprint tests: a photocell system (Kit Racetime 2 Light Radio, Microgate, Italy) was used to measure the time (s) needed to cover $10 \mathrm{~m}$ and $20 \mathrm{~m}$ distances. The starting point was $1 \mathrm{~m}$ before the position of the photocell so that each subject selected the moment of starting. The time was recorded from the moment when the participants intercepted the photocell beam. Each subject was allowed three attempts at the test and players received feedback of the score obtained in every sprint. The recovery time between attempts was at least $3 \mathrm{~min}$. The mean of the three test results was used for further analysis. Both absolute and partial reliability measures are presented in Table 1.

Vertical jump tests: the vertical jump height tests (i.e., CMJ and SJ) were performed using a jumping mat (Optojump, MicroGate, Italy), which measures the time the feet are off the mat and translates the result into a jump height in $\mathrm{cm}$. All participants were accustomed to both these tests. The technique was explained before each test and each athlete had 3 attempts at each jump. The SJ was started from a static semisquatting position with the knee angle of $90^{\circ}$ degrees of flexion, followed by subsequent action, during which the leg and hip extensor muscles contracted concentrically. At the beginning of the CMJ test, each participant stood erect on the jump mat. The test consisted of preparatory movement down to approximately $90^{\circ}$ degrees of knee flexion, stretching the leg extensor muscles followed by explosive maximal extension in the opposite direction. All subjects were informed of their performance. The recovery time between jumps was $3 \mathrm{~min}$. For the CMJ, the mean height of the last 2 attempts was included in the analysis while for the SJ, the mean of the last 3 attempts (i.e., repetitions 2 and 3 ) were used (Table 1).

Ball Release Velocity test: $\mathrm{BRv}$ was determined with a three-pace run up. The subject kept one foot on the ground at the moment of throwing. Each athlete used his dominant arm and personal technique. A standard handball was used (weight $480 \mathrm{~g}$, circumference $58 \mathrm{~cm}$ ). All subjects undertook 3 attempts with at least 2 min of recovery between attempts. Peak velocity was measured using a radar Stalker ATS gun (Radar Sales, Minneapolis, MMN, USA) positioned behind the goal. Just after each throw, the subject was informed of the velocity reached. The mean of repetitions 2 and 3 was entered in the analysis (Table 1).

Upper body force-velocity test: in the last testing session, athletes performed an incremental-load free bench press test (Salter). All players were accustomed to the bench press exercise. The bench position, barbell grip and displacement were previously established and controlled in each attempt or repetition. Participants performed a 15 min warm up and 2 sets of presses with a light load (i.e., barbell weight of $20 \mathrm{~kg}$ ) before starting the test protocol. The starting weight for the test was $25 \mathrm{~kg}$ and was increased by $10 \mathrm{~kg}$ up to a load of $85 \mathrm{~kg}$. The recovery time between sets was $4 \mathrm{~min}$. Three repetitions were performed at each intensity. During the incremental test, the descent phase of the bar was controlled by verbal instructions from 
the researcher as follows: "down" (2 s), "chest" (1 s), "go". To avoid the rebound effect, the "lift" signal was randomised. Then following the instructions of the investigator, each subject was encouraged to lift the barbell as quickly as possible during the concentric phase (i.e., explosively) until the elbows were completely extended. Strong verbal encouragement was used to motivate the participants. A linear displacement system was employed (T-Force System, Ergotech, Murcia, Spain) to record the mean velocity of the propulsive phase, mean power of the propulsive phase and the estimated 1 repetition maximum (1RMest). It had been shown that mean velocity could be used to precisely estimate loading intensity (González-Badillo and Sánchez-Medina, 2010). This device consists of a cable-extension connected to the barbell. Information of the movement velocity of the bar is transmitted and transformed from analogue to digital by a data acquisition board. The recorded data are analyzed by customized software. Vertical instantaneous velocity was directly sampled by the device at that the frequency of $1000 \mathrm{~Hz}$. The propulsive phase was defined as the portion of the concentric phase during which the measured acceleration (a) was greater than acceleration due to gravity (i.e., a $>-9.8 \mathrm{~m} \cdot \mathrm{s}^{-2}$ ) (González-Badillo and Sánchez-Medina, 2010). Once the test was completed and the data processed, the load corresponding to the mean maximum power (LoadMP) was determined for each individual. Then, for this load, we calculated the variables of mean power of the propulsive phase (PMPPMP) and the peak power (P्PeAKMP). The mean value of three repetitions was used as a final variable.

\section{Statistical Analyses}

All variables are provided as means \pm standard deviations (SD). The variables were transformed into the z-score in order to analyse the presence of outliers. The normal distribution of each variable was checked by the Shapiro-Wilk test. The intrasession Intraclass Correlation Coefficient was calculated for all tests (except for the 1RMest variable) using the equation 2.k (ICC2.k) (Weir, 2005). The coefficient of variation (CV) was calculated as $100\left(\mathrm{e}^{\mathrm{S}}-1\right)$ (Hopkins, 2011). Pearson correlation coefficients were used to determine interrelationships between variables. The strength of a correlation was defined as $\mathrm{r}=0-0.1$ (trivial), $\mathrm{r}=0.1-0.3$ (small), $\mathrm{r}=0.3-0.5$ (moderate), $\mathrm{r}=0.5$ -0.7 (large), $r=0.7-0.9$ (very large) and $r=0.9-1$ (nearly complete) (Hopkins, 2003). A repeated measures analysis of variance (RM ANOVA) was used to assess differences in the means recorded for the three repetitions of each dependent variable. When the sphericity test was violated, a Greenhouse-Geisser estimation was used to adjust the degree of freedom. The Bonferroni post hoc procedure was used to test multiple comparisons. Effect size was estimated using eta square partial $\left(\eta^{2} \mathrm{p}\right)$. The Pearson correlation coefficient was used as a measure of association between variables. We used hierarchical cluster analysis to create several groupings according to the results of the performance tests. Each variable was transformed into the z-score to avoid effects of different measurement scales. As a measure of similarity, squared Euclidean distances technique was used. The clustering Ward's method was used. A two between group multivariate analysis of variance (MANOVA) was used for upper trunk (1RMest, PPEAKMP, PMppMP and BRv) and lower trunk ( $\mathrm{ST}_{10}$, $\mathrm{ST}_{20}, \mathrm{CMJ}$ and SJ) variables, where the predictor variable was the cluster solution (i.e., Cluster 1, 2, 3 and 4). The players were classified into different clusters through a descriptive discriminant analysis. As the criterion variable, we used the 4cluster classification generated by the cluster analysis, while lower trunk ( $\mathrm{ST}_{10}, \mathrm{ST}_{20}, \mathrm{CMJ}$ and SJ) and upper trunk (1RMest, P PeakMP, PMpPMP and $\mathrm{BRv}$ ) variables were entered as the predictor variables. The level of significance was set at $p<$ .05. All statistical analyses were performed using SPSS software (SPSS Inc, Illinois, USA).

\section{Results}

Table 1 provides the means $( \pm \mathrm{SD})$, the $95 \%$ confident interval (CI) of the mean, $\mathrm{ICC}_{2 . \mathrm{k}}$ and $95 \%$ CI ICC $2 . k$ of the variables recorded (lower trunk [ST ${ }_{10}, \mathrm{ST}_{20}, \mathrm{CMJ}$ and SJ] and upper trunk [1RMest, PpeakMP, PmppMP and BRv]). In parallel, the 3 repetitions of each test and the variables examined were compared by RM ANOVA. Significant differences were detected for the CMJ $\left(\mathrm{F}[1.43,21.41]=2.2, p=.021, \eta^{2} \mathrm{p}=.13\right)$ and $\mathrm{BRv}(\mathrm{F}[2,30]$ $=8, p=.004, \eta^{2} \mathrm{p}=.35$ ); post hoc Bonferroni correction indicated differences between set 1 with $2(p=.01)$ for the CMJ variable and set 1 vs set $2(p=.020)$ and set 1 vs. set $3(p=.023)$ in BRv. Thus, we used the mean of set 2 and 3 for the final 
values of $B R v$ and the CMJ. For the rest of the variables the mean of the three attempts was used.

Pearson correlation coefficients between BRv and bench press variables (1RMest, P PeAKMP and PMPPMP) were respectively .68 $(p<.01)$ large, $.55(p<.05)$ large and $0.54(p<.05)$ large.

The hierarchical cluster analysis dendrogram constructed before examining any groupings is provided in Figure 1. In the analysis, each subject was first considered as an individual group i.e., 16 subjects $=16$ groups, in which each of the nine variables was analysed. Then, differences between all subjects were analysed with each subject assigned to the same group in a cluster matrix. We therefore selected 4 clusters.

After creating a grouping classification using a cluster analysis, the players were classified in 4 clusters. The means \pm SDs of all the variables of the grouped solution generated by the cluster analysis are provided in Table 2.

First multivariate statistical technique used (i.e., cluster analysis) provided a grouping classification of the players based on a measure of similarity (i.e., squared Euclidean distance) and the agglomeration method (i.e., Ward's method). This classification system was tested using a discriminant analysis. Before discriminant analysis, a MANOVA showed significant differences in the lower $\left(\lambda=.07 ; p=.004 ; \eta^{2} \mathrm{p}=.59\right)$ and upper trunk $\left(\lambda=.03 ; p=.0001 ; \eta^{2} \mathrm{p}=.69\right)$. Figure 2 shows $z$-score values of each variable (i.e., the upper and lower trunk) at each cluster solution obtained.

\begin{tabular}{|c|c|c|c|c|c|c|c|c|}
\hline & \multicolumn{8}{|c|}{$\begin{array}{l}\text { Table } 1 \\
\text { Descriptive variables recorded (provided as their mean, standard deviation, } \\
\text { Intraclass Correlation Coefficient }\left[I C C_{2 . k}\right], \text { Mean 95\% CI ICC } 2 . k \\
\text { and repeated measures analysis of variance [RM ANOVA]). }\end{array}$} \\
\hline & Mean \pm SD & $\begin{array}{l}\mathrm{CV} \\
(\%) \\
\end{array}$ & Min-Max & Mean CI (95\%) & ${ }^{*} \mathrm{ICC}_{2 . \mathrm{k}}$ & $\begin{array}{c}\mathrm{CI}(95 \%) \\
\mathrm{ICC}_{2 . \mathrm{k}} \\
\end{array}$ & SEM & $p$ \\
\hline $\mathrm{ST}_{10}(\mathrm{~s})$ & $1.69(0.085)$ & 4.5 & $1.56-1.81$ & $1.66-1.72$ & .72 & $.35-.89$ & .06 & .378 \\
\hline $\mathrm{ST}_{20}(\mathrm{~s})$ & $2.91(0.095)$ & 3.8 & $2.79-3.06$ & $2.86-2.96$ & .67 & $.25-.88$ & .09 & .062 \\
\hline $\mathrm{CMJ}(\mathrm{cm}) \#$ & $41.19(7.12)$ & 19 & $31.86-59.16$ & $37.39-44.98$ & .89 & $.68-.93$ & 3.37 & .165 \\
\hline $\mathrm{SJ}(\mathrm{cm})$ & $37.31(5.76)$ & 18.4 & $29.16-48.75$ & $34.24-40.12$ & .96 & $.92-.99$ & 1.98 & .161 \\
\hline $\operatorname{BRv}\left(m \cdot s^{-1}\right) \#$ & $27(1.52)$ & 6 & $24.72-29.31$ & $26.29-27.92$ & .93 & $.80-.98$ & .57 & .554 \\
\hline 1RMest $(\mathrm{kg})$ & $97(10.45)$ & 11 & $79-112$ & $91-109$ & $* *$ & $* *$ & $* *$ & $* *$ \\
\hline PpeakMP (W) & 953 (127) & 14.5 & $753-1021$ & $885-1021$ & .96 & $.90-.99$ & 34 & .365 \\
\hline PMPPMP (W) & $564(89)$ & 17 & $422-741$ & $516-612$ & .97 & $.92-.99$ & 21 & .442 \\
\hline * & $\begin{array}{c}=\text { intraclass c } \\
\text { sed. \# value u } \\
P_{M P P M P}= \\
1 R M_{\text {est }}= \\
S J=\text { squat }\end{array}$ & $\begin{array}{l}\text { rrelat } \\
\text { ed wa } \\
\text { PEAKN } \\
\text { nean } \\
\text { stima } \\
\text { ımp. }\end{array}$ & $\begin{array}{l}1 \text { coefficient } \\
\text { he mean of } r \\
=\text { peak powe } \\
\text { wer in a prop } \\
1 \text { repetition } \\
\text { IJ = countern } \\
\quad S T_{20}=2\end{array}$ & $\begin{array}{l}\left(C C_{2 . k}\right)^{* *}=\text { only } \\
\text { petitions } 2 \text { and } \\
\text { in the maximu } \\
\text { lsive phase in } \\
\text { maximum. BR } \\
\text { lovement jump. } \\
\text { m sprint time. }\end{array}$ & $\begin{array}{l}\text { one meas } \\
3 \text { (see pro } \\
n \text { power } \\
\text { te maxim } \\
=\text { ball re } \\
S T_{10}=10\end{array}$ & $\begin{array}{l}\text { ure of this vat } \\
\text { edures for de } \\
\text { ad. } \\
\text { im power loa } \\
\text { ease velocity. } \\
\text { sprint tim }\end{array}$ & & \\
\hline
\end{tabular}




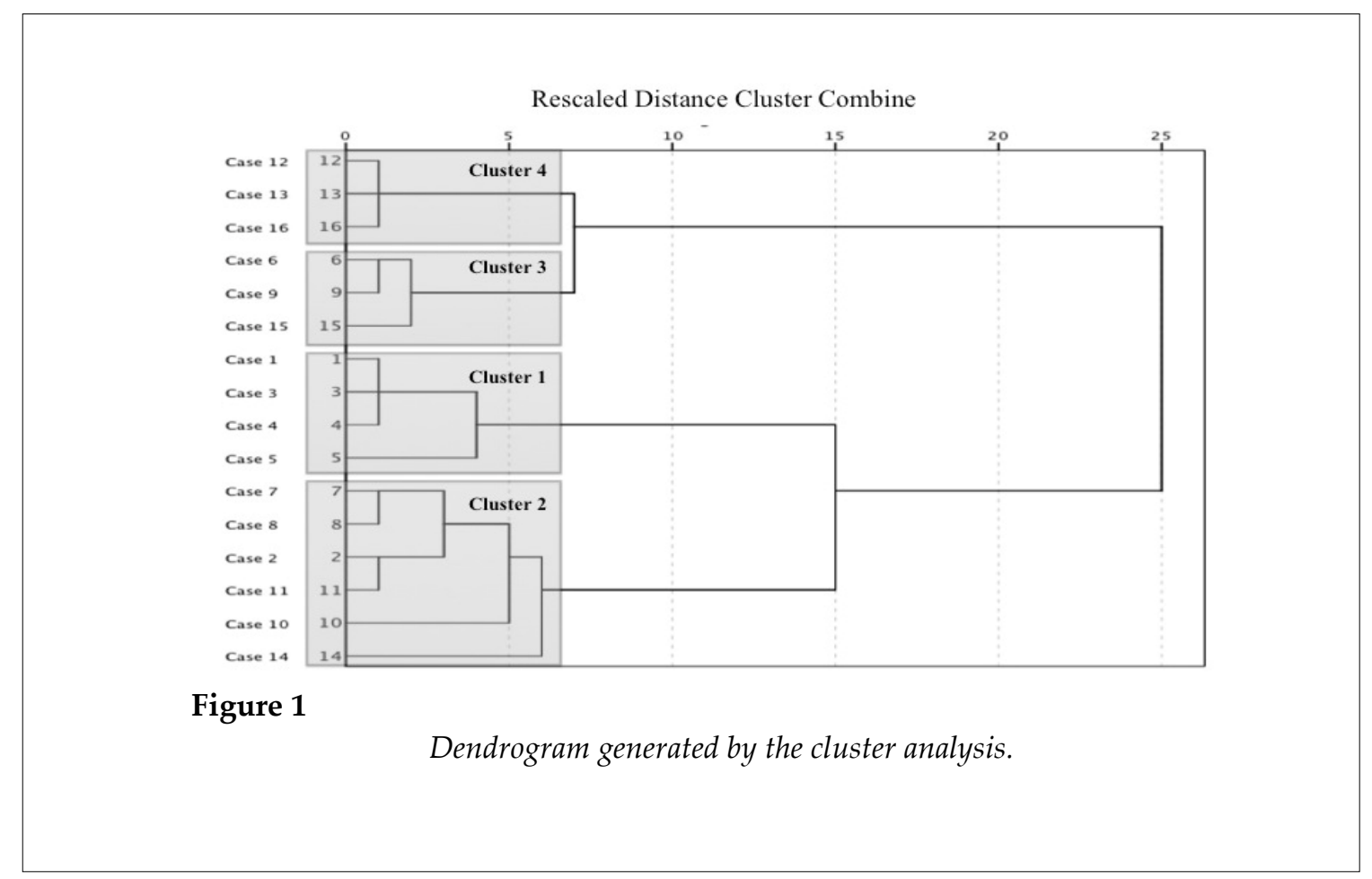

\begin{tabular}{|c|c|c|c|c|}
\hline \multicolumn{5}{|c|}{$\begin{array}{c}\text { Table } 2 \\
\text { Mean } \pm S D \text { of the performance indicators according to the clusters } \\
\text { generated by the hierarchical cluster analysis. }\end{array}$} \\
\hline \multicolumn{5}{|c|}{ Physical performance } \\
\hline & $\begin{array}{c}\text { Cluster 1 } \\
(\mathrm{n}=4)\end{array}$ & $\begin{array}{c}\text { Cluster } 2 \\
(\mathrm{n}=6)\end{array}$ & $\begin{array}{c}\text { Cluster } 3 \\
(\mathrm{n}=3)\end{array}$ & $\begin{array}{c}\text { Cluster } 4 \\
(\mathrm{n}=3)\end{array}$ \\
\hline $\mathrm{ST}_{10}(\mathrm{~s})$ & $1.68(.03)$ & $1.66(.63)$ & $1.76(.46)$ & $1.73(.03)$ \\
\hline $\mathrm{ST}_{20}(\mathrm{~s})$ & $2.90(.06)$ & $2.82(.04)$ & $3.01(.05)$ & $3.02(.05)$ \\
\hline CMJ (cm) & $47.92(7.65)$ & 42.49 (5.87) & $37.40(.45)$ & $33.12(2.28)$ \\
\hline $\mathrm{SJ}(\mathrm{cm})$ & $41.73(2.95)$ & $38.61(6.83)$ & $32.76(2.73)$ & $31.36(2.05)$ \\
\hline $\operatorname{BRv}\left(m \cdot s^{-1}\right)$ & $26.18(.88)$ & $28.68(.57)$ & $25.18(.68)$ & $27.13(.58)$ \\
\hline 1RMest (kg) & $89(6)$ & $104(6)$ & $85(7)$ & $104(7)$ \\
\hline PPEAKMP (W) & $808(60)$ & $1016(120)$ & $919(76)$ & $1053(63)$ \\
\hline $\operatorname{PMPPMP}(W)$ & $461(37)$ & $620(99)$ & $546(17)$ & $606(11)$ \\
\hline $\begin{array}{r}P \\
P_{M P P M P}= \\
1 R M_{\text {est }}=e \\
S J=\text { squat ju }\end{array}$ & $\begin{array}{l}\mathbf{M P}=\text { peak } p \\
\text { power in a } \\
\text { ited } 1 \text { repeti } \\
C M J=\text { coun } \\
\qquad T_{20}\end{array}$ & $\begin{array}{l}r \text { in the max } \\
\text { ulsive phase } \\
\text { maximum. } \\
\text { novement ju } \\
0 \text { m sprint ti }\end{array}$ & $\begin{array}{l}\text { um power } \\
\text { the maxim } \\
v=\text { ball rel } \\
S T_{10}=10\end{array}$ & $\begin{array}{l}\text { a power load. } \\
\text { se velocity. } \\
\text { sprint time. }\end{array}$ \\
\hline
\end{tabular}



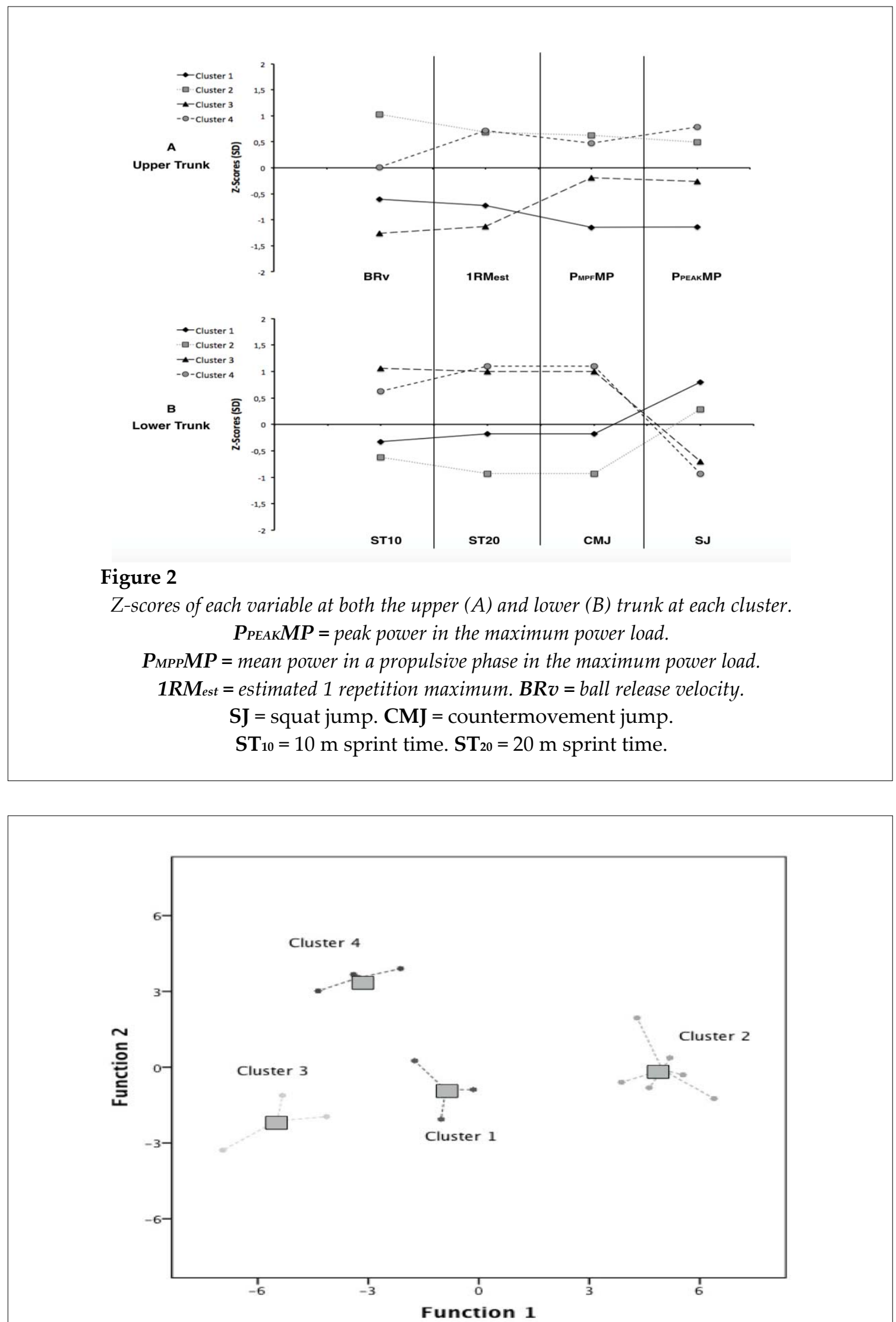

Figure 3

Graphical representation of each player (circle) and the centroid mean for each group (square). 
The following values were obtained from discriminant analysis: function $1(\lambda=.001 ; p=$ $.0001)$, function $2(\lambda=.044 ; p=.013)$ and function 3 $(\lambda=.24 ; p=.046)$. The percentage of variance explained by each of these functions was $75 \%$, $15 \%$ and $10 \%$, respectively. In other words, these three canonical functions explained $100 \%$ of variability between groups. Combined intragroup correlations between the discriminant variables and the typified canonical discriminant functions were $\mathrm{r}=.44$ and $-.39\left(\mathrm{BRv}\right.$ and $\mathrm{ST}_{20}$ for function 1 , respectively), $\mathrm{r}=.50$ (1RMest for function 2) and $\mathrm{r}=.48, \mathrm{r}=.47, \mathrm{r}=-.42,-.36$ and .22 (PMPFMP, PreakMP, CMJ, SJ and ST10 for function 3, respectively). Thus, discriminant analysis provided three canonical functions with specific weighted variables. These variables at each canonical function reflected the most important variable to classify players in the cluster solutions.

Discriminant analysis provided a probability of each player and their percentage probabilities of belonging to each cluster. This analysis revealed that $100 \%$ of cases were correctly classified in the 4-group solution generated by the cluster analysis using the performance variables assessed. All the players showed a $99 \%$ probability of belonging to a given cluster. Figure 3 shows the mean of the centroids (square) and the groupings of different players in their corresponding cluster (circle).

A qualitative analysis showed that players were distributed according to their playing positions as follows: cluster 1 (two center and two right back players); cluster 2 (one center, one left wing, two left backs, one right back and one pivot); cluster 3 (one goalkeeper, one right back and one left wing) and cluster 4 (two pivots and one goalkeeper).

\section{Discussion}

The aim of the present study was to identify different cluster groups of handball players according to their physical performance level assessed in a series of tests, which could then be used to design a training program based on the players individual needs. Moreover, our study sought to determine the variables that best reflected elite performance of a group of male handball players in the UI9 national team. To the best of our knowledge, this is the first attempt to create a classification scheme that analyses individual strengths and weaknesses in elite handball players based on their physical performance using a multivariate statistical approach.

Several studies have compared the performance of individual athletes considering a given skill or variable (e.g., BRv, $1 \mathrm{RM}$ in the bench press, RFD, repeated sprint ability) (Krüger et al., 2014; Marques et al., 2011; Michalsik et al., 2014). In competitive sports, physiologic variables interact and can obscure which factors are the real determinants of performance. In handball, factors such as BRv (Marques et al., 2007), training with external resistance (Hermassi et al., 2011) or combining different training modalities (van den Tillaar, 2004) seem to play an important role in the physical performance of players. In the present study, physical performance was assessed at two levels: the upper trunk (1RMest, P PeakMP, PMppMP and $\mathrm{BRv})$ and the lower trunk (CMJ, SJ, ST10 and $\mathrm{ST}_{20}$ ). Before classifying players according to their physical performance, a cluster analysis was performed to identify individuals obtaining similar scores in the performance tests used, i.e., according to their strengths and weaknesses. As it may be seen in the dendrogram generated by the cluster analysis (Figure 1), we opted for a 4-cluster solution. Table 2 provides the means and SDs of each performance variable analysed according to the cluster each player was assigned to. It is important to note that the player's classifications generate distinctive performance profiles. This information could be useful in order to create different training groups based on physical weakness points. In other words, using this statistical approach makes it possible to provide specific training to each group of players and enhance the physical performance of the players based on their specific needs. This allows for the identification of strengths and weaknesses in the three physical assessment dimensions (e.g., cluster 3 should focus on improving crosssectional and horizontal dimension performance and cluster 2 should improve vertical dimension performance) (Figure 2). Therefore, once the identification of strengths and weaknesses was performed, it was possible to design specific training sessions for each cluster solution.

It should be mentioned that the cluster analysis allocates the same weight to each performance variable examined. Thus, once all the 
players were grouped according to their physical performance, a discriminant analysis was used to determine whether the players could be classified in the established groups according to the performance variables tested and to check the specific weight of each variable for this purpose. This classification is graphically shown in Figure 3 , which represents the organization of the 16 players assessed. In Figure 3 it may be observed how the cluster analysis generated four extremely homogeneous groups in themselves, but these were heterogeneous compared to each other (each square represents the mean of the centroids of each group). In addition, discriminant analysis generated three discriminant canonical functions. Analysis of these functions demonstrated statistical significance in all cases when classifying clusters of players. Moreover, the first and second functions have greater discriminative power between players' classification $(75 \%$ and $15 \%$, respectively).

As it may be seen in the results' section, in the first canonical function (which was the function that explained more variance), BRv was the variable with most discriminatory power for the upper trunk, and ST20 for the lower trunk. Our results support these of previous literature suggesting that $\mathrm{BRv}$ is often considered as a key performance variable in handball players (Chelly et al., 2010; Hermassi et al., 2011; Marques and González-Badillo, 2006). In the second canonical discriminating function, $1 \mathrm{RM}$ est emerged as the variable with most statistical power. This variable has a lot of significance as several studies have shown the importance of maximum dynamic strength to improve $1 \mathrm{RM}$ est in handball players. Our results supported this relationship. A large correlation coefficient was obtained between $\mathrm{BRv}$ and 1 RMest $(\mathrm{r}=.68, \mathrm{p}<.01)$. As described before, it seems that these variables usually can be described by a positive linear function (Chelly et al., 2010; Marques, 2006; Marques and GonzálezBadillo, 2006). Finally, in the last canonical discriminatory function, the best performance indicators to correctly classify the players were PreakMP, PmppMP, CMJ, SJ and ST10. As mentioned earlier, no prior study had examined PPEAKMP and PMPPMP variable in handball players, although Marques et al. (2007) correlated the peak power attained during the bench press ( 26 and $36 \mathrm{~kg}$ ) with BRv $(r=.63, p=.017)$. However, in our study these variables (PMpPMP and PिеAKMP) returned a large coefficient with BRv $(\mathrm{r}=.55, \mathrm{p}<.05$ and $\mathrm{r}=$ $.54, \mathrm{p}<.05$, respectively).

The main limitation of our study was the sample size $(\mathrm{n}=16)$, although due to the selective nature of the elite population used, this was the maximum sample size available. However, our intention was not to generalize the results to the population, but to create a multivariate systematic tool to assess intra-squad physical performance. It can be therefore applied in order to acknowledge which variables require a training focus within the squad for certain individuals. It is interesting to note that this is the first study that uses a multivariate approach to analyse physical performance with a practical application. Information obtained in other age groups will help generate a broader database to classify potential players. Future systems, including more physiological and anthropometrical variables need to be developed. This will help identify new performance measures that will be able to better discriminate between players whose physical performance is optimal in a given dimension. Also future research needs to be conducted in order to make a cluster and discriminant analysis in the specific handball playing positions and moreover, including other types of variables (i.e., decision-making and endurance tests). This would allow identification of the most relevant strength training variables at each specific playing position.

The present study provides new information on specific interactions between physical performance and cluster created solutions. According to performance measured in upper body variables (1RMest, PreakMP, PMppMP and BRv) and others such as $10 \mathrm{~m}$ and $20 \mathrm{~m}$ sprint velocity, as well as CMJ and SJ height, we were able to classify 16 young male elite handball players into four groups through cluster analysis. The results revealed that within a young elite handball team there were various physical performance levels and therefore, these athletes required different training focuses based on their individual strengths and weaknesses. Finally, a discriminant analysis identified $\mathrm{BRv}, \mathrm{ST}_{20}, 1 \mathrm{RM}$ est, PpeakMP and PMppMP as the variables that best reflected elite physical performance in the handball players. 


\section{Practical implications}

The information emerging from this study can help researchers, coaches and strength and conditioning coaches objectively evaluate the results of physical fitness tests conducted on the players in their teams. Notably, the classification system obtained through cluster and discriminant analyses enabled us to discriminate four groups of athletes according to their performance considering several physical variables rather than just one (i.e., a multivariate approach). Using this method, players are assigned to clusters by physical variables that identify their strengths and weaknesses. As it can be seen in Figure 2, players assigned to cluster 3 were less physically fit and required improvement in the horizontal (e.g., ST10 and $\mathrm{ST}_{20}$ ) and cross-sectional dimensions (e.g., $1 R M$ est and BRv). To train the specific needs of this group, technique and maximum strength sessions could be implemented in their training schedule. Whereas, players assigned to cluster 1 needed enhancement in the cross-sectional dimension of the upper trunk only (i.e., P PEAKMP and PмpмМP). Training sessions based on high execution velocity would be a good choice for this cluster group to improve the variables related to muscular power. For the lower trunk, this could be in the form of plyometric training. Therefore, the cluster analysis allows coaches to group training sessions ensuring players will develop specific skills and abilities to enhance their physical performance parameters.

Finally, our discriminant analysis assigned greater relative weight to some of the performance variables assessed compared to others. Based on this type of objective quantifiable information, training sessions could be designed to improve performance in these specific physical measures.

\section{Acknowledgements}

The authors would like to thank the Spanish Handball Federation, especially the second coach (Javier Martín), the medical doctor (Jose Maria Calvillo) and David Reyero. Moreover, the main author is grateful to prof. Humberto Trujillo (Faculty of Psychology, University of Granada) for the advice in statistical analysis.

\section{References}

Baker J, Kungl AM, Pabst J, Straub B, Büsch D, Schorer J. Your fate is in your hands? Handedness, digit ratio (2D:4D), and selection to a national talent development system. Laterality, 2013; 18: 710-718

Bresciani G, Cuevas MJ, Garatachea N, Molinero O, Almar M, De Paz JA, Márquez S, González-Gallego J. Monitoring biological and psychological measures throughout an entire season in male handball players. Eu J Sports Sci, 2010; 10: 377-384

Chelly MS, Fathloun M, Cherif N, Ben Amar M, Tabka Z, Van Praagh E. Effects of a back squat training program on leg power, jump, and sprint performance in junior soccer players. J Strength Con Res, 2009; 23: 2241-2249

Chelly MS, Hermassi S, Aoudi R, Shephard RJ. Effects of 8 - weeks in-season plyometric training on upper and lower limb performance of elite adolescent handball players. J Strength Con Res, 2013; 28: 14011410

Chelly MS, Hermassi S, Shepard RJ. Relationship between power and strength of the upper and lower limb muscle and throwing velocity in male handball players. J Strength Con Res, 2010; 24: 1480-1487

Gabbett TJ, Jenkins DG, Abernethy B. Relative importance of physiological, antropometric, and skill qualities to team selection in professional rugby league. J Sports Sci, 2011; 29: 1453-1461

Gabbett TJ. A comparison of physiological and anthropometric characteristics among playing positions in sub-elite rugby league players. J Sports Sci, 2006; 24: 1273-1280

González-Badillo JJ, Sánchez-Medina L. Movement velocity as a measure of loading intensity in resistance training. Int J Sports Med, 2010; 31: 347-352

Gorostiaga EM, Granados C, Ibáñez J, González-Badillo JJ, Izquierdo M. Effects of an Entire Season on Physical Fitness Changes in Elite Male Handball Players. Med Sci Sports Exerc, 2005; 38: 357-366

Gorostiaga EM, Izquierdo M, Iturralde P, Ruesta M, Ibañez J. Effects of heavy resistance training on maximal 
and explosive force production, endurance and serum hormones in adolescent handball players. Eur J Appl Occup Physiol, 1999; 80: 485-493

Granados C, Izquierdo M, Ibañez J, Bonnabau EM, Gorostiaga EM. Differences in physical fitness and throwing velocity among elite and amateur female handball players. Int J Sports Med, 2007; 28: 860-867

Granados C, Izquierdo M, Ibáñez J, Ruesta M, Gorostiaga EM. Effects of an entire season on physical fitness in elite female handball players. Med Sci Sports Exerc, 2008; 40: 351-361

Hermassi S, Chelly MS, Tabka Z, Shephard RJ, Chamari K. Effects of 8-week in-season upper and lower limb heavy resistance training on the peak power, throwing velocity, and sprint performance of elite male handball players. J Strength Con Res, 2011; 29: 2424-2433

Hoare DG. Predicting success in junior elite basketball players-the contribution of antropometric and physiological attributes. J Sci Med Sport, 2000; 3: 391-405

Hopkins WG. A scale of magnitudes for effect statistics In: A New View of Statistics; 2003. Available at: URL: http://sportsci.org/resource/stats/effectmag.html; accessed on: 11.11.2014

Hopkins WG. Precision of measurement. In: A New View of Statistics; 2011. Available at URL_ http://newstats.org/precision.html; accessed on: 11.11.2014

Ingebrigtsen J, Jeffreys I, Rodahl S. Physical characteristics and abilities of junior elite male and female handball players. J Strength Con Res, 2013; 27: 302-309

Krüger K, Pilat C, Ückert K, Frech T, Mooren FC. Physical performance profile of handball players is related to playing position and playing class. J Strenght Con Res, 2014; 28: 117-125

Lidor R, Falk B, Arnon M, Cohen Y, Segal G, Lander Y. Measurement of talent in team handball: the questionable use of motor and physical tests. J Strength Con Res, 2005; 19: 318-325

Marques MC, González-Badillo JJ. In-Season Resistance Training and Detraining in Professional Team Handball. J Strength Cond Res, 2006; 20: 563-571

Marques MC, Saavedra FJ, Abrantes C, Aidar FJ. Associations between rate of force development metrics and throwing velocity in elite team handball players: a short research report. J Hum Kinet, 2011; 29: 5357

Marques MC, van den Tillaar R, Vescovi JD, González-Badillo JJ. Relationship between throwing velocity, muscle power, and bar velocity during bench press in elite handball players. Int J Sports Physol Perform, 2007; 2: 414-422

Marques MC. In-Season Strength and Power Training for Professional Male Team Handball Players. Strength Cond J, 2010; 32: 74-81

Matthys SPJ, Vaeyens R, Vandendriessche J, Vandorpe B, Pion J, Coutts AJ, Lenoir M, Philippaerts RM. A multidisciplinary identification model for youth handball. Eu J Sports Sci, 2011; 11: 355-363

Michalsik LB, Madsen K, Aagaard P. Match performance and physiological capacity of female elite team handball players. Int J Sports Med, 2013; 35: 595-607

Michalsik LB, Madsen K, Aagaard P. Physiological capacity and physical testing in male elite handball. $J$ Sports Med Phys Fitness, 2015; 55: 415 - 429

Mohamed H, Vaeyens R, Matthys S, Multail M, Lefevre J, Lenoir M, Philppaerts R. Anthropometric and performance measures for the development of a talent detection and identification model in youth handball. J Sports Sci, 2009; 27: 257-266

Peterson MD, Rhea MR, Alvar BA. Maximizing strength development in athletes: a meta-analysis to determine the dose-response relationship. J Strength Cond Res, 2004; 18: 377-382

Póvoas SCA, Ascensao AAMR, Magalhaes J, Saebra AF, Krustrup P, Soares JMC, Rebelo ANC. Physiological demands of elite team handball with special reference to playing position. J Strength Con Res, 2014; 28: $430-442$

Rhea MR, Alvar BA, Ball SD. A meta-analysis to determine the dose response for strength development. Med Sci Sports Med, 2003; 35: 456-464 
Unnithan V, White J, Georgiu A, Iga J, Drust B. Talent identification in youth soccer. J Sports Sci, 2012; 30: 1719-1726

van Der Tillar R. Effect of different training programs on the velocity overarm throwing: a brief review. $J$ Strength Con Res, 2004; 18: 388-396

Vila H, Manchado C, Rodriguez N, Abraldes JA, Alcaraz PE, Ferragut C. Anthropometric profile, vertical jump, and throwing velocity in elite female handball players by playing positions. J Strength Con Res, 2012; 26: 2146-2155

Votteler A, Höner O. The relative age effect in the German Football TID programme: Biases in motor performance diagnostics and effects on single motor abilities and skills in groups of selected players. Eur J Sport Sci, 2013; 14: 433-442

Weir P. Quantifying test-retest reliability using the intraclass correlation coefficient and the SEM. J Strength Cond Res, 2005; 19: 231-240

William AM, Reilly T. Talent identification and development in soccer. J Sports Sci, 2000; 18: 657-667.

\section{Corresponding author:}

\section{Iker J. Bautista}

FisioSalud Elite, Health, Training \& Innovation

Calle Alicante, no13 (18210) Peligros, Granada

Phone (H): +34 663962174

E-mail: ikerugr@gmail.com 